\title{
The choreography of cyclooxygenases in the kidney
}

Commentary

See related article, pages 61-69.

\author{
G.A. FitzGerald
}

Center for Experimental Therapeutics, School of Medicine, 153 Johnson Pavillion, 3620 Hamilton Walk, University of Pennsylvania, Philadelphia, Pennsylvania 19104-6084, USA. Phone: (215) 898-1184; Fax: (215) 573-9135; E-mail: garret@spirit.gcrc.upenn.edu.

J. Clin. Invest. 110:33-34 (2002). doi:10.1172/JCI200216044.

\author{
Nibil est ab omni \\ parte beatum. \\ (No blessing is unmixed.) \\ -Horace
}

Consideration of the cardiovascular effects of cyclooxygenase (COX) inhibition has generated much heat and some light since the emergence of selective inhibitors of COX2 onto the US market in January 1999. COX2 inhibitors have gained an increasingly dominant share of the prescription market for nonsteroidal anti-inflammatory drugs (NSAIDs), currently dividing yearly sales of over $\$ 6$ billion among three products: celecoxib, rofecoxib, and valdecoxib.

Approval of these first three coxibs was based on their superiority over conventional NSAIDs (which inhibit both COX1 and COX2) in surrogate studies of endoscopically visualized gastric ulceration. Gastric epithelial COX1 is thought to generate the prostaglandins (PGs) - $\mathrm{PGE}_{2}$ and prostacyclin $\left(\mathrm{PGI}_{2}\right)$ - that afford cytoprotection, while $\mathrm{COX} 2$ is thought to be the dominant source of PGs - interestingly, also $\mathrm{PGE}_{2}$ and $\mathrm{PGI}_{2}$ - that mediate pain and inflammation (1). The results of the Vioxx Gastrointestinal Outcomes Research (VIGOR) study (2) verified the COX2 hypothesis. More than 8,000 patients, mostly with rheumatoid arthritis, were randomized to receive therapeutically equivalent doses of rofecoxib or naproxen, a traditional NSAID. The incidence of prespecified primary and secondary clinical endpoints reflecting ulceration and its complications was significantly reduced, from around $4 \%$ on naproxen to around $2 \%$ on the COX 2 inhibitor. Perhaps surprisingly, the incidence of cardiovascular events diverged between the groups, with an almost fivefold difference in the incidence of myocardial infarction.

\section{Cardiovascular complexities}

Speculation as to whether this difference reflected chance, a cardioprotective benefit of naproxen, a cardiovascular hazard of the coxib, or a coincident effect of both drugs has been as animated and prolonged as it has been inconsequential. Meanwhile, competitive claims have emerged around the issue of renovascular hypertension, a recognized adverse effect of traditional NSAIDs. There is a paucity of well-controlled studies of the impact of coxibs on blood pressure. However, the incidence of hypertension, crudely reported as a serious adverse effect during drug development, appears to relate to coxib dose and to increase as a function of drug selectivity.

The PG system is a reactive one, highly conditioned by context. Thus, inhibition of PG formation, either by NSAIDs, aspirin, or COX2 inhibitors, is remarkably well tolerated by otherwise healthy individuals. Similarly, despite effects of PGs on platelet function and vascular tone in vitro, mice lacking the relevant $P G$ receptors rarely exhibit a cardiovascular phenotype, unless manipulated in some manner. For example, deletion of the IP gene, encoding the receptor for the vasodilating platelet inhibitory $\mathrm{PGI}_{2}$, does not result in thrombosis but rather increases the response to prothrombotic stimuli (3). Similarly, $\mathrm{PGI}_{2}$ modulates the evoked response to the vasoconstrictor platelet agonist $\mathrm{TxA}_{2}$ in vivo (4). However, although selective COX2 inhibitors depress $\mathrm{PGI}_{2}$ without coincident inhibition of COX1-derived $\mathrm{TxA}_{2}(5)$, this would only represent a theoretical hazard in those otherwise at risk for thrombosis. Perhaps patients with rheumatoid arthritis constituted such a population in the VIGOR study (6).

The salt and water retention and hypertension that complicate PG suppression in the kidney seem to teach a similar lesson. COX inhibition does not normally influence blood pressure, but it may sensitize an animal to agents that do. IP knockout mice are not hypertensive, but they exhibit an enhanced pressor response to salt loading. Mice lacking the EP2 receptor for $\mathrm{PGE}_{2}$ are also normotensive, but their pressor responses to salt loading or infusion of the vasoconstrictor angiotensin II (AII) are heightened (7). Furthermore, the ability to elaborate vasodilator PGs, such as $\mathrm{PGE}_{2}$ and $\mathrm{PGI}_{2}$, becomes critical to the maintenance of renal blood flow under renoprival conditions, such as might result from activation of the renin/angiotensin or sympathoadrenal systems $(8,9)$. Examples might include patients with severe heart failure or athletes subject to dehydration during strenuous exercise. In part, this reflects how PGs generally function as cardiovascular counterligands. Thus, products of platelet activation, such as $\mathrm{TxA}_{2}$, evoke COX2-dependent endothelial release of $\mathrm{PGI}_{2}$ in vitro (10), and systemic $\mathrm{PGI}_{2}$ biosynthesis is elevated, along with $\mathrm{TxA}_{2}$, in syndromes of accelerated platelet-vessel wall interactions (11). Similarly, local infusion of a vasoconstrictor, such as AII, evokes vascular release of $\mathrm{PGI}_{2}$, which modulates its effect on vascular tone in humans (12). Individual PGs may contribute to this buffering interplay. Thus, $\mathrm{PGE}_{2}$ activates both dilator EP2 receptors and constrictor $\mathrm{EP} 3$ receptors in the murine vasculature (13). Thus, COX products have divergent effects on vascular tone and might be expected either to modulate or amplify the effects of vasoconstrictors, such as AII.

\section{Roles of the COX isoforms in regulating vascular tone}

Breyer and colleagues have pursued the delicate renal choreography of the COXs in this issue of the JCI (14). Data from $C O X$ gene deletion studies and selective inhibition of COX1 and 
COX2 are consistent and suggest that COX products differentially modulate the pressor response to infused AII in the mouse. Vasoactivity of the products appears to segregate with $\mathrm{COX}$ isoform; $C O X-2$ deletion or inhibition amplifies the pressor response to AII, whereas COX-1 deletion or inhibition largely abolishes the response to the vasoconstrictor. Blockade or deletion of COX2 also reveals a depressive effect of AII on medullary blood flow, which may account for the papillary necrosis sometimes seen as a complication of NSAID administration. Given the prior indication that $\mathrm{COX} 2$ is a dominant source of systemic $\mathrm{PGI}_{2}$ formation (5), the more regulated nature of COX2 expression (1), and the predominance of this isoform in the medulla, these latter results are, perhaps, unsurprising. If all the renovascular complications of NSAIDs were attributable to COX2 inhibition, this would be consistent with reports of similar renovascular adverse effect profiles for the coxibs and their NSAID comparators in arthritis trials $(15,16)$.

At first glance, a contrasting role for COX1-dependent constrictor prostanoids may seem more provocative. However, Coffman and colleagues have previously shown that COX1 deficiency, like AII deficiency, causes natriuresis and that COX1 deletion enhances the effects of angiotensinconverting enzyme (ACE) inhibitors. Indeed deletion of the AT1A receptor for AII amplifies the effect on blood pressure and sodium sensitivity of knocking out COX1 (17). Furthermore, Nasjletti has described how AII prompts release of vascular $\mathrm{TxA}_{2}$ and how $\mathrm{TxA}_{2}$ receptor (TP) antagonists lower blood pressure in rats with AIIdependent hypertension (18). AII also activates vascular NADPH oxidasedependent free radical generation (19). Certain isoprostanes, free radical-catalyzed PG isomers, may contribute to TP activation in vivo (20). The mechanisms that underlie the segregation of function and subcellular compartmentalization of the COXs in cells that express both isoforms is poorly understood (21). However, consistent with the present observations of Qi et al. (14), COX1 preferentially couples with Tx and PGF synthase in coexpression systems in vitro, while COX2 couples functionally with PGI synthase (22).

All of these findings might suggest that the incidence of renovascular complications of traditional NSAIDs and selective COX2 inhibitors might diverge in favor of the cheaper drugs, but this does not appear to be the case $(15,16)$. Perhaps the mice do not simulate faithfully the human condition. Indeed, several issues constrain our willingness to extend these observations to humans: the animals were anesthetized; only females were studied; most of the findings derive from a single model, that of short-term responses to a single pressor agonist; and the dosing regimens of the pharmacological probes were not assessed rigorously for selectivity in vivo.

Nonetheless, the choreography of COXs in humans, no less than in mice, is likely to be conditioned by context. Both the acute thrombotic and renovascular profiles of coxibs are reassuringly bland in the large numbers of patients at low risk who have been studied to date. However, just as the coxibs have been administered to few populations at increased risk of thrombosis, so have they been studied in few individuals with renoprival syndromes, such as those with cardiac, hepatic, or renal failure with complicating secondary hyperaldosteronism. These and previous results in mice (17) raise the possibility that both the increasing degrees of COX2 inhibition and the selectivity with which this inhibition is attained in the renal compartment are important. Both relate to the incidence of renovascular complications in predisposed individuals. Detailed studies in humans will be necessary to address this hypothesis.

1. FitzGerald, G.A., and Patrono, C. 2001. The cox ibs, selective inhibitors of cyclooxygenase-2. N. Engl. J. Med. 345:433-442.

2. Bombardier, C., et al. 2000. Comparison of upper gastrointestinal toxicity of rofecoxib and naproxen in patients with rheumatoid arthritis. N. Engl. J. Med. 343:1520-1528.

3. Murata, T., et al. 1997. Altered pain perception and inflammatory response in mice lacking prostacyclin receptor. Nature. 388:678-682.
4. Cheng, Y., et al. 2002. Role of prostacyclin in the cardiovascular response to thromboxane $\mathrm{A}_{2}$. Science. 296:539-541.

5. McAdam, B., et al. 1999. Systemic biosynthesis of prostacyclin by cyclooxygenase (COX)-2: the human pharmacology of a selective inhibitor of COX-2. Proc. Natl. Acad. Sci. USA. 96:272-277.

6. DeMaria, A.N. 2002. Relative risk of cardiovascular events in patients with rheumatoid arthritis. Am. J. Cardiol. 39:33D-38D.

7. Kennedy, C.R., et al. 1999. Salt-sensitive hypertension and reduced fertility in mice lacking the prostaglandin EP2 receptor. Nat. Med. 5:217-220.

8. Data, J.L., Chang, L.C., and Nies, A.S. 1976. Alteration of canine renal vascular response to hemorrhage by inhibitors of prostaglandin synthesis. Am. J. Physiol. 230:940-945.

9. Jackson, E.K., Gerkens, J.F., Brash, A.R., and Branch, R.A. 1982. Acute renal artery constriction increases renal prostaglandin $\mathrm{I}_{2}$ biosynthesis and renin release in the conscious dog. J. Pharmacol. Exp. Ther. 222:410-413.

10. Caughey, G.E., Cleland, L.G., Gamble, J.R., and James, M.J. 2001. Up-regulation of endothelial cyclooxygenase- 2 and prostanoid synthesis by platelets. Role of thromboxane A2. J. Biol. Chem. 276:37839-37845.

11. FitzGerald, G.A., Smith, B., Pedersen, A.K., and Brash, A.R. 1984. Increased prostacyclin biosynthesis in patients with severe atherosclerosis and platelet activation. N. Engl. J. Med. 310:1065-1068.

12. Broughton Pipkin, F., Morrison, R., and O'Brien, P.M. 1989. Prostacyclin attenuates both the pressor and adrenocortical response to angiotensin II in human pregnancy. Clin. Sci. (Lond.) 76:529-534.

13. Audoly, L.P., et al. 2001. Role of EP2 and EP3 $\mathrm{PGE}_{2}$ receptors in control of murine renal hemodynamics. Am. J. Physiol. Heart Circ. Physiol. 280:H327-H333.

14. Qi, Z., et al. 2002. Opposite effects of cyclooxyge nase- 1 and -2 activity on the pressor response to angiotensin II. J. Clin. Invest. 110:61-69. doi:10.1172/JCI200214752.

15. Whelton, A., Maurath, C.J., Verburg, K.M., and Geis, G.S. 2000. Renal safety and tolerability of celecoxib, a novel cyclooxygenase- 2 inhibitor. $A m$. J. Ther. 7:159-157.

16. Gertz, B.J., Krupa, D., Bolognese, J.A., Sperling, R.S., and Reicin, A. 2002. A comparison of adverse renovascular experiences among osteoarthritis patients treated with rofecoxib and comparator non-selective non-steroidal antiinflammatory agents. Curr. Med. Res. Opin. 18:82-91.

17. Athirakul, K., Kim, J.S., Audoly, L.P., Smithies, O., and Coffman, T.M. 2001. Deficiency of COX-1 causes natriuresis and enhanced sensitivity to ACE inhibition. Kidney Int. 60:2324-2329.

18. Nasjletti, A. 1998. Arthur C. Corcoran Memorial Lecture. The role of eicosanoids in angiotensindependent hypertension. Hypertension. 31:194-200.

19. Griendling, K.K., Sorescu, D., and Ushio-Fukai, M. 2000. NADPH oxidase: role in cardiovascular biology and disease. Circ. Res. 86:494-501.

20. Audoly, L.P., et al. 2000. Cardiovascular responses to the isoprostanes $\mathrm{iPF}_{2 \alpha}$-III and $\mathrm{IPE}_{2}$-III are mediated via the thromboxane $\mathrm{A}_{2}$ receptor in vivo. Circulation. 101:2833-2840.

21. Smith, W.L., and Langenbach, R. 2001. Why there are two cyclooxygenase isozymes. J. Clin. Invest. 107:1491-1495.

22. Ueno, N., et al. 2001. Coupling between cyclooxygenase, terminal prostanoid synthase, and phospholipase A2. J. Biol. Chem. 276:34918-34927. 